\title{
28 Research Suare \\ Experimental Validation of a Potential Circulating microRNA Panel for Non-Invasive Pancreatic Cancer Diagnosis
}

\section{Ali Seyed Salehi}

Shahid Beheshti University of Medical Sciences

\section{Negar Parsa}

Islamic Azad University

Farnaz Roshan-Farzad

Islamic Azad University

Ali Behmanesh

Iran University of Medical Sciences

Mohadeseh Fathi

Shahid Beheshti University of Medical Sciences

Hamid Asaszadeh aghdaei

Shahid Beheshti University of Medical Sciences

Roshanak Shams ( $\nabla$ shams.rosha.86@gmail.com )

Iran University of Medical Sciences

\section{Research Article}

Keywords: MicroRNA, Pancreatic cancer, Diagnostic Panel

Posted Date: October 4th, 2021

DOl: https://doi.org/10.21203/rs.3.rs-936432/v1

License: (9) (i) This work is licensed under a Creative Commons Attribution 4.0 International License. Read Full License 


\section{Abstract}

MicroRNA (miRNA) expression dysregulations in pancreatic ductal adenocarcinoma (PDAC) have been studied widely for their diagnostic and prognostic utility. By the use of Bioinformatics-based methods, in our previous study, we identified some potential miRNA panels for diagnosis of Pancreatic cancer patients from non-cancerous controls (the screening stage). In this report, we used 142 plasma samples from people with and without pancreatic cancer (PC) to conduct RT-qPCR differential expression analysis to assess the strength of the first previously proposed diagnostic panel (consisting of miR-125a-3p, miR4530 and miR-92a-2-5p). For this aim the research was divided into two phases: testing, and external validation. A total of 92 PC plasma samples and normal controls (NCs) were evaluated in the testing stage to determine the ability of the considered miRNAs to discriminate cancer from non-cancer samples as a panel. Furthermore, in the external validation phase, a group of 25 PC serum samples vs. 25 NCs was employed to validate the diagnostic value of the panel. As the result, we identified significant upregulation for all the three considered miRNAs in the serum of PC patients. After that, a three-miRNA panel in serum was developed. For the testing, validating and combined stages, the area under the receiver operating characteristic curves (AUC) for the panel were $0.850,0.910$ and 0.86 respectively, indicating that it had a higher diagnostic value than individual miRNAs. Therefore, we detected a promising three-miRNA panel in the plasma for non-invasive PC diagnosis (miR-125a-3p, miR-4530 and miR-92a-2-5p).

\section{Introduction}

According to statistics from patients in England and Wales, pancreatic cancer (PC) has the lowest overall survival rate of any cancer kind, with even less than $1 \%$ overall 10 -year survival and $3 \%$ overall 5 -year survival (1). In most cancers Surgical resection is the only way to cure the cancer, but most PC patients are asymptomatic until it's too late, and they may lose out on the greatest chance for a clinical procedures (2). Existing diagnostic procedures, such as imaging technologies and tumor biopsy, nevertheless have drawbacks, including causing further damages, being more expensive, and having lower sensitivity and specificity (3). As a result, effective and noninvasive screening approaches are in highly demanded in order to enhance overall PC survival. During the last decade biomarker-based techniques have been proven to be effective in the early diagnosis of variety of cancers (4). Meanwhile, the sole FDA-approved biomarker for PC diagnosis is (CA19-9), however its indiscriminate expression in other conditions such as false-positive elevation in the context of obstructive jaundice have severely hampered its practical application in PC diagnosis (5). As a result, finding new tumor biomarkers that can provide more robust and trustworthy diagnostic information for PC detection is crucial.

MicroRNAs are a type of non-coding RNA that regulate gene expression by degrading mRNA or inhibiting translation (6). In the context of cancer, tumor suppressor miRNAs and oncogenic miRNAs are two main types of miRNAs that have a role in carcinogenesis. The role of the target mRNAs in the tumor start pathway determines the classification (7-9). Because miRNA expression profiles differ significantly across cancer types, miRNA expression profiles could well be employed as a promising non-invasive 
diagnostic tool (10). A rising number of research have focused on circulating miRNAs as noninvasive biomarkers for cancer diagnosis in recently. Numerous circulating miRNAs have been described by several researchers as putative biomarkers for $P C(8,11,12)$. In our previous study, By the use of Bioinformatics-based methods, we identified some potential miRNA panels for diagnosis of Pancreatic cancer patients from non-cancerous controls (13). Overall, 5 diagnostic models based on specified expression algorithms and functional features of miRNAs were proposed, each comprising of different combinations of miRNAs. In this study by focusing on the first proposed panel consisting of miR-125a$3 p$, miR-4530, and miR-92a-2-5p, we aimed to analyze the expression of these miRNAs in plasma samples of 142 people with and without pancreatic cancer through RT-qPCR method. To evaluate the considered panel two phases of validations were done. Then we evaluated the diagnostic value of those miRNAs as a unified diagnostic panel. Ultimately, this kind of novel circulating miRNA biomarkers with high specificity and sensitivity for the early detection of PC are hoped to be applicable after more clinical evaluations.

\section{Material And Methods}

Ethics statement. All study was conducted in compliance with applicable guidelines and regulations. All subjects signed an informed consent form. The Iran National Committee for Ethics in Biomedical Research approved the research (IR.SBMU.RIGLD.REC.1398.031).

Patients. This research included 71 histopathologically confirmed PC patients and also 70 non-cancerous donors who had been referred for clinical checkup from 2017 to 2020. all participants were selected from Gastroenterology clinic, Taleghani Hospital, Shahid Beheshti University of Medical Sciences, Tehran, Iran. Prior to surgery and/or chemotherapy, blood samples were collected from subjects. For 47 PC Patients, clinical characteristics such as gender, age, FBS, tobacco smoking and alcohol addiction, disease history, CA199 level, etc. were available and documented (Supplementary Table 1). $5 \mathrm{ml}$ of peripheral venous blood was taken in tubes containing EDTA and after half an hour of incubation at room temperature, centrifuged at $4000 \mathrm{rpm}$ for 10 minutes. Plasma samples were then collected in $1 \mathrm{ml}$ aliquots in polypropylene tubes with special lids and stored at $-80^{\circ} \mathrm{C}$ until further biochemical analysis.

Study design. Study design. As the screening and training phase of our research had been already done through comprehensive bioinformatics methods in our previous study (13), we divided this study into two phases validation procedure (testing and validating) and a final specific bioinformatics evaluation of the 3 considered miRNAs. In addition, because the demographics and clinicopathological characteristics of 50 of our Pancreatic Cancer patients were accessible, the relationships between these variables and the expression levels of the miRNAs under consideration were also investigated.

Total RNA extraction. Total RNA was isolated from $200 \mathrm{~L}$ of human plasma using the Trizole reagent (Invitrogene) Isolation Kit according to the manufacturer's protocol.

CDNA synthesis and RT-qPCR. The expression analysis of miRNAs was performed using BON-MiR specific PCR kits. In this study, specific stem-loop primers were used to synthesize CDNA for each miRNA. 
The microRNA expression analysis was performed using a BON miRNA detection kit provided by Stem Cell Technology Company (BN-0011.17). This kit uses SYBR® Green in a simple two-step PCR method to measure all desired RNAs. Briefly, the reverse transcription reaction was performed in $20 \mu \mathrm{L}$ mixture containing $4 \mu \mathrm{L}$ of RNA extracted from the plasma, $1 \mu \mathrm{L}$ BON-RT adaptor $(10 \mu \mathrm{M}), 2 \mu \mathrm{L}$ of $10 \mathrm{mM}$ dNTPs, $4 \mu \mathrm{L}$ of RT buffer, $1.0 \mu \mathrm{L}$ of AMV reverse transcriptase and, $8 \mu \mathrm{L}$ of DEPC-treated water. The tubes were incubated at $25^{\circ} \mathrm{C}$ for 10 minutes, $42^{\circ} \mathrm{C}$ for 60 minutes, and $70^{\circ} \mathrm{C}$ for 10 minutes for CDNA synthesis. Subsequently the following polymerase chain reactions (PCR) were performed: 1 cycle of $95^{\circ} \mathrm{C}$ for 2 minutes, followed by 40 cycles of $95^{\circ} \mathrm{C}$ for 5 seconds and, $60^{\circ} \mathrm{C}$ for 30 seconds using a Corbett Research RotorGene machine. The $2-\Delta \Delta \mathrm{Ct}$ approach was used to measure the relative expression levels of miRNAs to the reference miRNA (has-miR-24).

Statistical analysis. The unpaired student T-test was used to compare serum miRNA expression levels in PC patients and non-cancer controls. To correlate demographic and clinical characteristics between classes and their relationship with miRNA expression levels, an oneway ANOVA or chisquared test was used. Receiver operating characteristic (ROC) curves and the area under the ROC curve (AUC) were used to assess the diagnostic performance of the detected serum miRNAs and panels. A multiple logistic model was developed using the four miRNAs. The RStudio software version 1.2.5033 for windows and GraphPad Prism 8.0 (GraphPad Software, USA) were applied for data analysis and graph drawing. A twosided $\mathrm{P} 0.05$ was regarded as statistically significant.

\section{Results}

RT-qPCR evaluation of proposed miRNAs. For the testing phase, we used RT-qPCR to assess the levels of miRNA expression in 52 PC plasma samples vs 40 NCs. Interestingly, we found that miR-125a-3p and miR-4530 and miR-92a-2-5p expression levels were significantly up-regulated in plasma from PC patients compared with that from in noncancerous controls. The expression of these miRNAs have also been evaluated by qRT-PCR in an external validation cohort of 25 PC plasma samples vs $25 \mathrm{NCs}$, and finally in the whole combinations of samples. When the testing and validating phases were combined, the three miRNAs exhibited the same upregulated expression pattern as when the two phases were separated $(\mathrm{P}>0.05$ ) (Table1 and Figure 1 ). We also performed Pearson correlation analysis between demographics data and the expression of considered miRNAs to assess potential correlations of some clinical parameters such as gender, age, smoking, drinking, diabetes mellitus, and etc. Except for a reverse correlation between Alkaline phosphatase level with the expression level of miR-4530 ( $r=-0.416, p<0.001)$, none of the other variables had a significant effect on serum miRNA expression in $P C$ patients $(P>0.05$; data not shown), demonstrating the integrity of the miRNA signatures discovered.

Diagnostic performance of the 3-miRNAs panel in plasma. To assess the diagnostic significance of each of the six selected miRNAs, we used ROC analysis and computed AUC values. The AUCs for miR-125a-3p, miR-4530, miR-92a-2-5p in the combined cohort of the 
testing and validating phases were 0.853 (95 \% CI: $0.746-0.921$; sensitivity $=86.8 \%$, specificity $=68.8 \%), 0.764(95 \%$ CI: 0.615-0.854; sensitivity $=81.5 \%$, specificity $=64 \%)$ and 0.743 (95 \% CI: 0.686-0.831; sensitivity $=80 \%$, specificity $=68.8 \%$ ) respectively (Table 2 and Figure 2). Furthermore, we established a 3-miRNA panel by combining the three serum miRNAs and testing its diagnostic performance for PC comparing to a single miRNA first in testing, validating and then the combined cohorts. In a logistic regression model, the predicted probability of PC diagnosis by the panel was computed using the following formula: $\operatorname{Logit}(\mathrm{P})=-12.6+8.69 \times \operatorname{miR}-125 \mathrm{a}+5.7 \times \operatorname{miR}-4530+8.7 \times \operatorname{miR}-92 \mathrm{a}-2-5 \mathrm{p}$. The panel's ROC analysis was also performed for all testing, validating and combining stages and the AUCs were 0.850, 0.910 and 0.86 respectively (Figure 3). Figure 4 demonstrates the prediction power of the combined panel in discriminating PC from controls.

Bioinformatics evaluation of the three proposed miRNAs. Based on analytically established miRNA functions, we used MiRNet (https://www.mirnet.ca/), an online miRNA pathway analysis and systems biology web-tool, to interpret the functional characteristics of the 3 miRNAs (14). Target genes prediction was performed using miRTarbase platform for each of the specified miRNAs. A general network demonstrating all interaction between miRNAs and the target genes was prepared (Figure 5A) and then, amongst in common target genes of the considered miRNAs were extracted to form a final module consisting of 27 nodes and 48 edges (Figure 5B). Table 3 and 4 show the results of pathway analysis utilizing the Kyoto Encyclopedia of Genes and Genomes (KEGG) and Gene Ontology (GO) analyses. Mir125a, Mir-92a-2-5p, and Mir-4530 were shown to have 310, 247, and 134 target genes, respectively. There were 17 target genes in common between miR-125a-3p and miR-92a-25 p. 5 and 2 other target genes were also in common between miR-125a-3p, miR-4530 and miR-92a-2-5p. Figure 5B demonstrates the interactions between those genes. KEGG analysis showed that the most 3 significant pathway correlated with this genes are TGFbeta signaling pathway, Colorectal cancer and MAPK signaling pathway. GO analysis also showed that Negative regulation of signal transduction, response to carbohydrate stimulus, response to drug and Notch signaling pathway were the most significant terms.

\section{Discussion}

PC has caused a serious health risk to people all around the world in recent decades, with a fatality rate approximately equal to the incidence rate (15). To some degree, new early detection methods appear to be more effective in improving overall prognosis for PC patients than new therapy drugs (16). The Food and Drug Administration has approved CA19.9 as the sole biomarker for clinical PC management (17, 18). However, because CA19.9 levels rise in other malignancies and even benign conditions, it has little practical utility as a PC diagnostic biomarker (19). A growing number of novel biomarkers or molecular targets for PC diagnosis, such as circulating miRNAs, have developed in recent years. Many miRNAs can serve as promising biomarkers for numerous diseases, including cancer, because of their stable existence in peripheral circulation $(8,20)$. 
In our previous study, as the discovery phase, we combined several serum expression profiles of miRNAs to discover the most significant miRNA signatures helpful in PC diagnosis and then created innovative miRNA diagnosis models for PC using multiple bioinformatics methods. In the training phase, based on the area under the curve (AUC) scores, 27 differentially expressed miRNAs were selected. Afterwards, five diagnostic models comprising of distinct combinations of miRNAs, based on their significant expression algorithms and functional features, were introduced using multivariate cox regression analysis. In this study, we focused on the first previously proposed panel to assess the expression levels and diagnostic performance of miR-125a-3p, miR4530 and miR-92a-2-5p as a panel in plasma samples from pancreatic cancer patients and non-cancerous controls. For this aim a two-phase analysis was carried out to discover differential expression of considered miRNAs in plasma samples from PC and non-cancerous patients. In total, we analyzed 77 PC plasma samples vs 65 NCs for the testing and validation phases. RTqPCR was used to assess miRNA signatures. As a result, we were able to confirm that PC had significantly higher levels of miR-125a-3p, miR-4530, and miR-92a-2-5p versus NC plasma samples. Regarding decrease or increase of expression patterns, this discovery does not appear to be consistent with the results of our previous bioinformatics analyses, but we should take into account that the literature has published controversial statements about the expression of these miRNAs in PC or other types of malignancies. On the other hand, preliminary research suggests that expression profiles of miRNAs derived from clinical samples such as blood or tissue vary depending on the assay method (microarray, PCR, sequencer) (21). Aside from that, we evaluated the expression of the considered miRNAs in an Iranian population as a separate genetics pool using a different way of analysis such as RT-qPCR, which is a more reliable method than Microarray. In a study by Kojima et al. (21), as well as our prior bioinformatics investigations, miR-125a-3p was found to be down-regulated in plasma samples of PC patients. Several other researches have also reported the down-regulation of this miRNA in plasma and tumor tissues of PC patients, according to the literature (22-24). However, our search for has-miR125a-3p and Pancreatic cancer in the database of Differentially Expressed MiRNAs in Human Cancers (dbDEMC) (25) revealed that the expression of this miRNA was also up-regulated in two other studies (GSE71533 and GSE74562). Little is known about the expression levels of miR-92a-2-5p and miR-4530 in pancreatic cancer, despite the fact that some studies have reported contradictory results regarding the expression of this genes in pancreatic cancer and also other types of cancers $(21,25-28)$. Our Roc curve analyses also revealed that when used as a single marker, miR-125a-3p, miR-92a-2-5p, and miR-4530 had $85.3 \%, 74.3 \%$, and $76.4 \%$ accuracies in distinguishing PC from non-cancerous controls, respectively. We had hoped to improve the diagnostic performance by combining three miRNAs to test and validate one of the panels that we had previously shown (13). We finally achieved a more robust discriminant performance with a sensitivity of $80.4 \%$, a specificity of $87.2 \%$, and an accuracy of $86.2 \%$ in detecting pancreatic cancer vs healthy controls by combining three miRNAs. Even though we showed that a combo of these three serum miRNAs can be used to discriminate PC, the biological functions of those miRNAs are still being debated. To that end, we conducted another round of bioinformatics analyses to assess the functional roles of the miRNAs under consideration in interactions with their target genes. The prediction of miRNA target genes revealed that these three miRNAs share some target genes. Figure $5 \mathrm{~A}$ depicts the entire network of miRNA target genes and the patterns of their interactions with one another. KEGG 
pathway analysis of common target genes revealed that these genes are mostly involved in TGFbeta/signaling, Colorectal cancer, and MAPK signaling pathways. TGF signaling plays a dual role in pancreatic cancer, promoting and inhibiting it depending on the stage of the disease. Some studies have shown that TGF- $\beta$ promotes apoptosis and inhibits cell cycle progression through $\mathrm{G} 1$ arrest, which has a potent antiproliferative activity in early stage pancreatic cancer $(29,30)$. However, some other studies have found that TGF- $\beta$ stimulates pancreatic cancer invasion and metastasis during the advanced stages of carcinogenesis (31). TGF-signaling therapeutic approaches have already shown usefulness in pancreatic cancer for both preclinical and clinical trials (30). We identified three target genes (RHOA, MYC and THBS1) implicated in this pathway which may be targeted by miR-125a-3p and miR-92a-2-5p (Table 3). Regarding pancreatic cancer tumorigenesis, studies have demonstrated that overexpression of RHOA motivates pancreatic cancer cells to invade and migrate (32). MiRNAs has been reported to prevent the activation of human pancreatic stellate cells (hPSCs) by targeting thrombospondin 1 (THBS1) and the downstream TGF-b pathway, according to some studies (33). Among the genes mentioned, MYC is likely the most important in the carcinogenesis of pancreatic cancer. Several studies have reported the overexpression of this gene in PC and some resources proposed MYC-targeted therapy as an effective method for PC treatment $(34,35)$. The results of $G O$ analysis also showed that Negative regulation of signal transduction, response to carbohydrate stimulus and response to drug are the most significant terms amongst the target genes of considered miRNAs, Most of them correlated to tumorigenesis of pancreatic cancer directly or non-directly $(12,36,37)$. Regarding the direct correlation of miR-4530 expression with serum alkaline phosphatase levels that we discovered, we should note that some studies have introduced elevated serum alkaline phosphatase levels as a marker of poor prognosis of pancreatic cancer (38)(39).

Theoretically, cancer cells are thought to leak exosomes into the circulation, which contain numerous molecular markers such as proteins, DNA, and miRNAs, and transport them to distant parts of the body in order to form a new environment for future invasion (40). The differential expression of certain MiRNAs in tumor tissue samples from cancer patients should be in concordance with sera from the same patients, according to this concept; nevertheless, some research did not find this to be the case (41). As a result, other biological pathways for the secretion of circulating miRNAs should be addressed. Our findings showed that plasma samples from PC patients had up-regulation of miR-125a-3p, miR-92a-2-5p, and miR-4530, despite the fact that some researches had found down-regulation of these genes (particularly for miR-125-3p) in plasma or tissues from PC patients and introduced these miRNAs with tumor suppressive role. However, the change of this miRNAs in some other studies has produced mixed results. Even if the down-regulated levels of these miRNAs in plasma are linked to particular specific oncogenes involved in PC carcinogenesis, their role in PC has been described and has to be investigated further. In this paper, we present the first evidence of a link between increased plasma levels of miR-125a-3p, miR92a-2-5p, and miR-4530 in PC patients. Much more importantly, our findings reveal that serum miRNAs, specifically a diagnostic score based on the combination of three predictive miRNAs, may identify patients with pancreatic tumors when compared to non-cancerous healthy controls. We believe that diagnosing pancreatic cancer with circulating markers in blood, which are relatively easy to obtain from 
most patients, is of higher quality, especially as an initial screening test. Overall, our study found new diagnostic circulating miRNAs in PC, while more researches with larger sample size is needed to confirm the diagnostic performance of the identified miRNAs in PC.

\section{Declarations}

\section{Ethics statement}

All study was conducted in compliance with applicable guidelines and regulations. All subjects signed an informed consent form. The Central Iran Region Committees on Health Research Ethics approved the research (IR.SBMU.RIGLD.REC.1398.031).

\section{Author Contributions}

RS proposed the study and wrote the manuscript and performed the bioinformatics analyses. AS performed the experimental tests and gathered the data. AB performed the statistical analysis. NP, MF and FR contributed to the collection of the biological sample. HA clinically revised the manuscript. All the authors reviewed and approved the final draft.

\section{Funding}

The study was supported by Research Institute for Gastroenterology and Liver Diseases, Shahid Beheshti University of Medical Sciences, Tehran, Iran

\section{Conflict of Interest}

The authors declare that the research was conducted in the absence of any commercial or financial relationships that could be construed as a potential conflict of interest.

\section{References}

1. Daoud, A. Z., Mulholland, E. J., Cole, G. \& McCarthy, H. O. MicroRNAs in Pancreatic Cancer: Biomarkers, prognostic, and therapeutic modulators. BMC cancer, 19 (1), 1-13 (2019).

2. Kamisawa, T., Wood, L. D., Itoi, T. \& Takaori, K. Pancreatic cancer. The Lancet, 388 (10039), 73-85 (2016).

3. Costa-Silva, B. et al. Pancreatic cancer exosomes initiate pre-metastatic niche formation in the liver. Nature cell biology, 17 (6), 816-826 (2015).

4. Crawley, A. S. \& O’Kennedy, R. J. The need for effective pancreatic cancer detection and management: a biomarker-based strategy. Expert Review of Molecular Diagnostics, 15 (10), 1339 1353 (2015).

5. Marrelli, D. et al. CA19-9 serum levels in obstructive jaundice: clinical value in benign and malignant conditions. The American journal of surgery, 198 (3), 333-339 (2009). 
6. Hill, C. G., Jabbari, N., Matyunina, L. V. \& McDonald, J. F. Functional and evolutionary significance of human microRNA seed region mutations. PLoS One, 9 (12), e115241 (2014).

7. Iorio, M. V. \& Croce, C. M. MicroRNA dysregulation in cancer: diagnostics, monitoring and therapeutics. A comprehensive review. EMBO molecular medicine, 4 (3), 143-159 (2012).

8. Savareh, B. A. et al. A machine learning approach identified a diagnostic model for pancreatic cancer through using circulating microRNA signatures., 20 (6), 1195-1204 (2020).

9. Shams, R. et al. MicroRNAs targeting MYC expression: trace of hope for pancreatic cancer therapy. a systematic review. Cancer management and research, 12, 2393 (2020).

10. Acunzo, M., Romano, G., Wernicke, D. \& Croce, C. M. MicroRNA and cancer-a brief overview. Advances in biological regulation, 57, 1-9 (2015).

11. Morimura, R. et al. Novel diagnostic value of circulating miR-18a in plasma of patients with pancreatic cancer. British journal of cancer, 105 (11), 1733-1740 (2011).

12. Fathi, M., Ghafouri-Fard, S., Abak, A. \& Taheri, M. Emerging roles of miRNAs in the development of pancreatic cancer. Biomed. Pharmacother, 141, 111914 (2021).

13. Shams, R. et al. Identification of potential microRNA panels for pancreatic cancer diagnosis using microarray datasets and bioinformatics methods. Scientific reports, 10 (1), 1-15 (2020).

14. Chang, L., Zhou, G., Soufan, O. \& Xia, J. miRNet 2.0: network-based visual analytics for miRNA functional analysis and systems biology. Nucleic acids research, 48 (W1), W244-W51 (2020).

15. Bray, F. et al. Global cancer statistics 2018: GLOBOCAN estimates of incidence and mortality worldwide for 36 cancers in 185 countries. CA: a cancer journal for clinicians, 68 (6), 394-424 (2018).

16. Del Chiaro, M., Segersvärd, R., Lohr, M. \& Verbeke, C. Early detection and prevention of pancreatic cancer: is it really possible today? World journal of gastroenterology: WJG, 20 (34), 12118 (2014).

17. Ballehaninna, U. K. \& Chamberlain, R. S. The clinical utility of serum CA $19-9$ in the diagnosis, prognosis and management of pancreatic adenocarcinoma: An evidence based appraisal. Journal of gastrointestinal oncology, 3 (2), 105 (2012).

18. Partyka, K., Maupin, K. A., Brand, R. E. \& Haab, B. B. Diverse monoclonal antibodies against the CA 19-9 antigen show variation in binding specificity with consequences for clinical interpretation., 12 (13), 2212-2220 (2012).

19. Singh, S., Tang, S., Sreenarasimhaiah, J., Lara, L. F. \& Siddiqui, A. The clinical utility and limitations of serum carbohydrate antigen (CA19-9) as a diagnostic tool for pancreatic cancer and cholangiocarcinoma. Digestive diseases and sciences, 56 (8), 2491-2496 (2011).

20. Mitchell, P. S. et al. Circulating microRNAs as stable blood-based markers for cancer detection. Proceedings of the National Academy of Sciences. 2008;105(30):10513-8.

21. Kojima, M. et al. MicroRNA markers for the diagnosis of pancreatic and biliary-tract cancers. PloS one, 10 (2), e0118220 (2015). 
22. Ling, S. et al. CircRHOT1 mediated cell proliferation, apoptosis and invasion of pancreatic cancer cells by sponging miR-125a-3p. Journal of Cellular and Molecular Medicine, 24 (17), 9881-9889 (2020).

23. Moriya, C. et al. Inhibition of PRDM14 expression in pancreatic cancer suppresses cancer stem-like properties and liver metastasis in mice., 38 (6), 638-648 (2017).

24. Yan, Q. et al. The serum microrna signatures for pancreatic cancer detection and operability evaluation. Frontiers in bioengineering and biotechnology, 8, 379 (2020).

25. Yang, Z. et al. dbDEMC 2.0: updated database of differentially expressed miRNAs in human cancers. Nucleic acids research, 45 (D1), D812-D8 (2017).

26. Lv, H. et al. MicroRNA-92a promotes colorectal cancer cell growth and migration by inhibiting KLF4. Oncology research, 23 (6), 283 (2016).

27. Elshafei, A., Shaker, O., Abd El-motaal, O. \& Salman, T. The expression profiling of serum miR-92a, miR-375, and miR-760 in colorectal cancer: An Egyptian study. Tumor Biology, 39 (6), 1010428317705765 (2017).

28. Zhang, G. et al. MicroRNA-92a functions as an oncogene in colorectal cancer by targeting PTEN. Digestive diseases and sciences, 59 (1), 98-107 (2014).

29. Hanahan, D. \& Weinberg, R. A. Hallmarks of cancer: the next generation. cell, 144 (5), 646-674 (2011).

30. Shen, W. et al. -q. TGF- $\beta$ in pancreatic cancer initiation and progression: Two sides of the same coin. Cell \& bioscience 2017;7(1):1-7.

31. Kano, M. R. et al. Improvement of cancer-targeting therapy, using nanocarriers for intractable solid tumors by inhibition of TGF- $\beta$ signaling. Proceedings of the National Academy of Sciences. 2007;104(9):3460-5.

32. Guo, X. et al. Balanced Tiam1-rac1 and RhoA drives proliferation and invasion of pancreatic cancer cells. Mol. Cancer Res, 11 (3), 230-239 (2013).

33. Asama, $\mathrm{H}$. et al. MicroRNA let-7d targets thrombospondin-1 and inhibits the activation of human pancreatic stellate cells., 19 (1), 196-203 (2019).

34. Hessmann, E., Schneider, G., Ellenrieder, V. \& Siveke, J. MYC in pancreatic cancer: novel mechanistic insights and their translation into therapeutic strategies., 35 (13), 1609-1618 (2016).

35. Wirth, M., Mahboobi, S., Krämer, O. H. \& Schneider, G. Concepts to target MYC in pancreatic cancer. Molecular cancer therapeutics, 15 (8), 1792-1798 (2016).

36. Yeh, J. J. \& Der, C. J. Targeting signal transduction in pancreatic cancer treatment. Expert opinion on therapeutic targets, 11 (5), 673-694 (2007).

37. Wu, Y-M., Nowack, D. D., Omenn, G. S. \& Haab, B. B. Mucin glycosylation is altered by proinflammatory signaling in pancreatic-cancer cells. Journal of proteome research, 8 (4), 1876-1886 (2009). 
38. Ohno, I. et al. Clinical significance of serum alkaline phosphatase level in advanced pancreatic cancer. Journal of Clinical Oncology, 29 (4_suppl), 183-183 (2011).

39. Xiao, Y. et al. Dynamic serum alkaline phosphatase is an indicator of overall survival in pancreatic cancer. BMC cancer, 19 (1), 1-8 (2019).

40. Challagundla, K. B. et al. microRNAs in the tumor microenvironment: solving the riddle for a better diagnostics. Expert review of molecular diagnostics, 14 (5), 565-574 (2014).

41. Bookland, M., Gillan, E., Song, X. \& Kolmakova, A. Peripheral circulation miRNA expression of pediatric brain tumors and its relation to tumor miRNA expression levels. Journal of Neurosurgery: Pediatrics, 26 (2), 136-144 (2020).

\section{Tables}




\begin{tabular}{l|l|l|l}
\hline \multicolumn{1}{|c|}{ MiRNA } & miR-125a-3p & miR-4530 & miR-92a-2-5p \\
\hline P-value & $<0.0001$ & 0.001 & $<0.0001$ \\
\hline $\begin{array}{l}\text { Differences } \\
\text { between means } \\
\pm \text { SEM }\end{array}$ & $0.23 \pm 0.0241$ & $0.186 \pm 0.036$ & $0.178 \pm 0.02$ \\
\hline AUC & 0.85 & 0.76 & 0.73 \\
\hline Specificity & 68.8 & 64 & 68.4 \\
\hline Sensitivity & 86.8 & 81.5 & 80.1 \\
\hline P-value & $<0.0001$ & $<0.0001$ & $<0.0001$ \\
\hline
\end{tabular}

Table 2 Tabular view displaying performance of combined panel of miR-125a-3p, miR-92a-25p and miR-4530

\begin{tabular}{|c|c|c|c|c|}
\hline Combinations & AUC & SE & SP & Opt Cutoff \\
\hline Combined cohort & 0.862 & 0.804 & 0.872 & 0.577 \\
\hline Testing & 0.851 & 0.811 & 0.85 & 0.574 \\
\hline Validating & 0.91 & 0.863 & 0.84 & 0.607 \\
\hline
\end{tabular}

Table 3 KEGG pathway analysis results

\begin{tabular}{|c|c|c|c|c|}
\hline Pathway & $\overline{\text { Tota }}$ & Expected & Hits & Pval \\
\hline TGF-beta/signaling pathway & 84 & 0.197 & 3 (RHOA, MYC, THBS1) & 0.00083 \\
\hline Colorectal cancer & 49 & 0.115 & 2 (RHOA, MYC) & 0.00556 \\
\hline MAPK signaling pathway & 265 & 0.623 & 3 (ARRB1, MYC, STK4) & 0.0211 \\
\hline $\begin{array}{l}\text { Leukocyte/transendothelial } \\
\text { migration }\end{array}$ & 108 & 0.254 & 2 (RHOA, MSN) & 0.0253 \\
\hline Pathways in cancer & 310 & 0.729 & 3 (RHOA, MYC, STK4) & 0.0319 \\
\hline Wnt signaling pathway & 144 & 0.338 & 2 ((RHOA, MYC) & 0.0431 \\
\hline
\end{tabular}


Table 4 GO-BP term analysis results

\begin{tabular}{|c|c|c|c|c|}
\hline GO Term & Total & Expected & Hits & Pval \\
\hline Negative/regulation/of/signal/transduction & 790 & 1.27 & $\begin{array}{l}\text { 6(RHOA, ARRB1, MYC, } \\
\text { THBS1, PHLDA3, STK4) }\end{array}$ & 0.00126 \\
\hline response to carbohydrate stimulus & 143 & 0.23 & 3 (RHOA, ARRB1, THBS1) & 0.0015 \\
\hline response to drug & 344 & 0.554 & 4 (RHOA, MYC, THBS1, PFAS) & 0.00204 \\
\hline Notch signaling pathway & 177 & 0.285 & 3 (ARRB1, MYC, AGO2) & 0.00276 \\
\hline cell-cell junction organization & 186 & 0.299 & 3 (RHOA, CDH6, THBS1) & 0.00317 \\
\hline regulation of binding & 189 & 0.304 & 3 (ARRB1, THBS1, SUMO1) & 0.00332 \\
\hline negative regulation of response to stimulus & 967 & 1.56 & $\begin{array}{l}\text { 6(RHOA, ARRB1, MYC, } \\
\text { THBS1, PHLDA3, STK4) }\end{array}$ & 0.00352 \\
\hline positive regulation of translation & 56 & 0.0901 & 2 (RHOA, THBS1) & 0.00362 \\
\hline Negative/regulation/of/myeloid/cell/differentiation & 60 & 0.0966 & 2 (MYC, TOB2) & 0.00415 \\
\hline $\begin{array}{l}\text { transforming growth factor beta receptor signaling } \\
\text { pathway }\end{array}$ & 221 & 0.356 & 3 (RHOA, MYC, THBS1) & 0.00514 \\
\hline regulation of translation & 228 & 0.367 & 3 (RHOA, THBS1, AGO2) & 0.00561 \\
\hline
\end{tabular}

\section{Figures}

\section{Differential Expression}

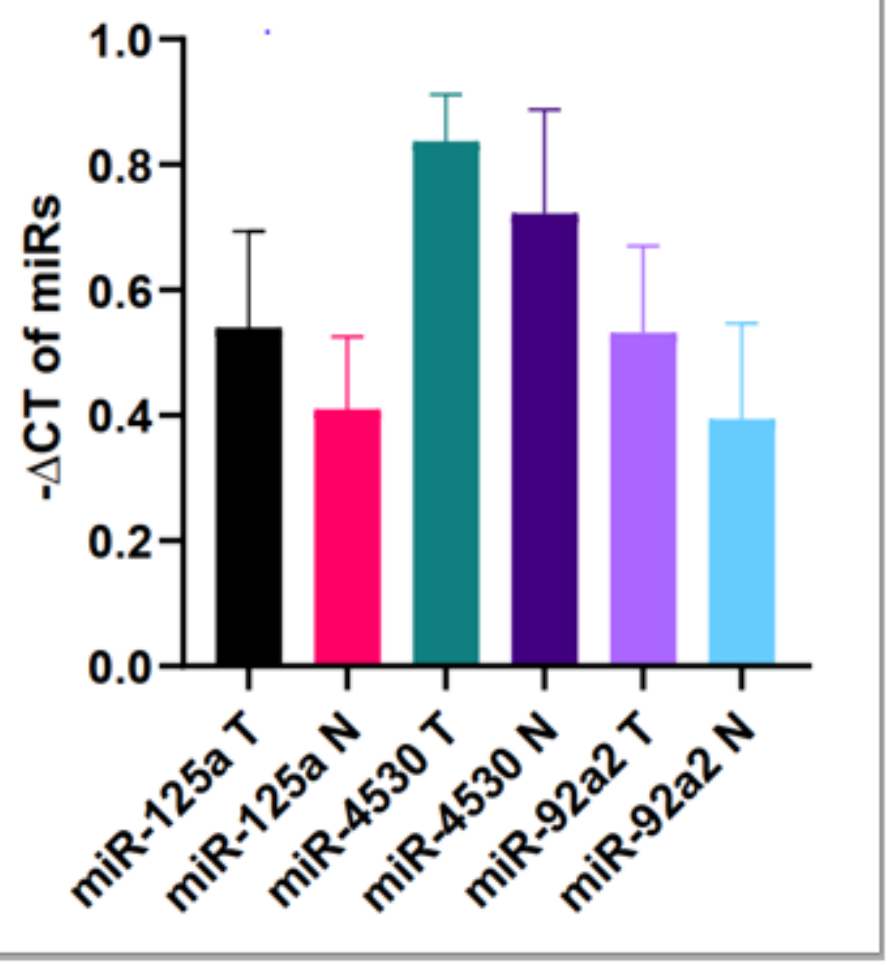


Figure 1

the differential expression values chart of circulating levels of miRNAs in healthy subjects and in patients with pancreatic cancer. All data was normalized to 0-1 scale

ROC curve: ROC of ${ }^{\times *} \mathrm{miR}-125 a-3 p$

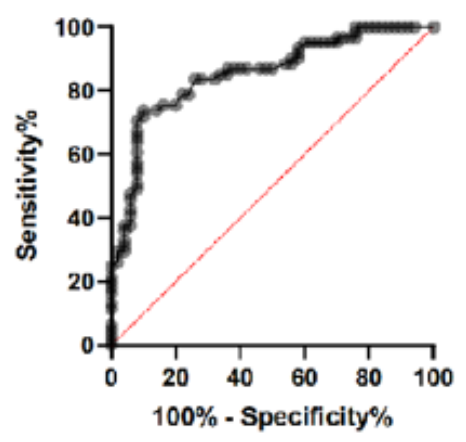

ROC curve: ROC of **miR-4530

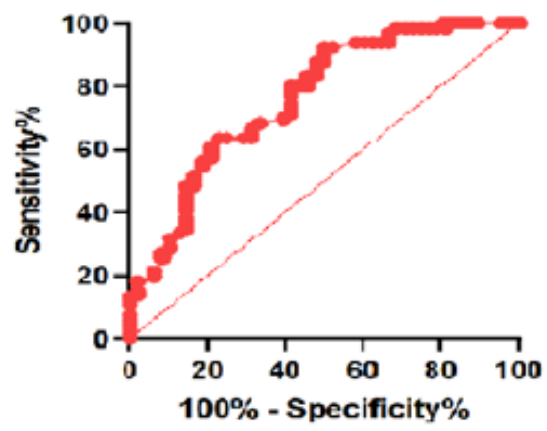

ROC curve: ROC of **miR-92a-2

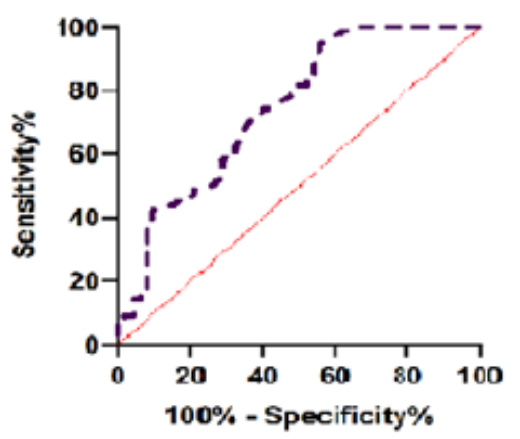

Figure 2

Receiver operating characteristics (ROC) curve analysis of miR-125a-3p and miR-4530 and miR-92a-2-5p in discriminating patients with pancreatic cancer from non-cancerous subjects.

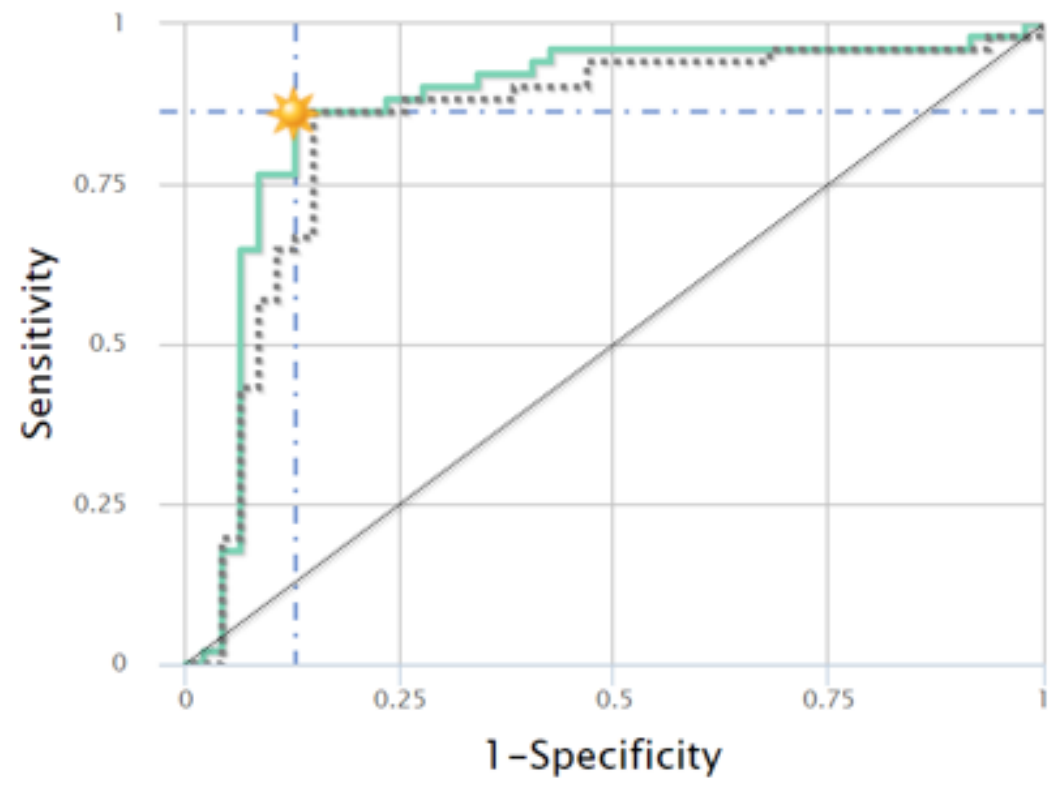

- Combo III * 10-fold CV, Combo III 301 Opt Cutoff 90.577

- diagonal line

\section{Figure 3}

ROC analysis of a combination of four miRNAs (miR-125a-3p, miR-4530 and miR-92a-2-5p) 


\section{Predictions}

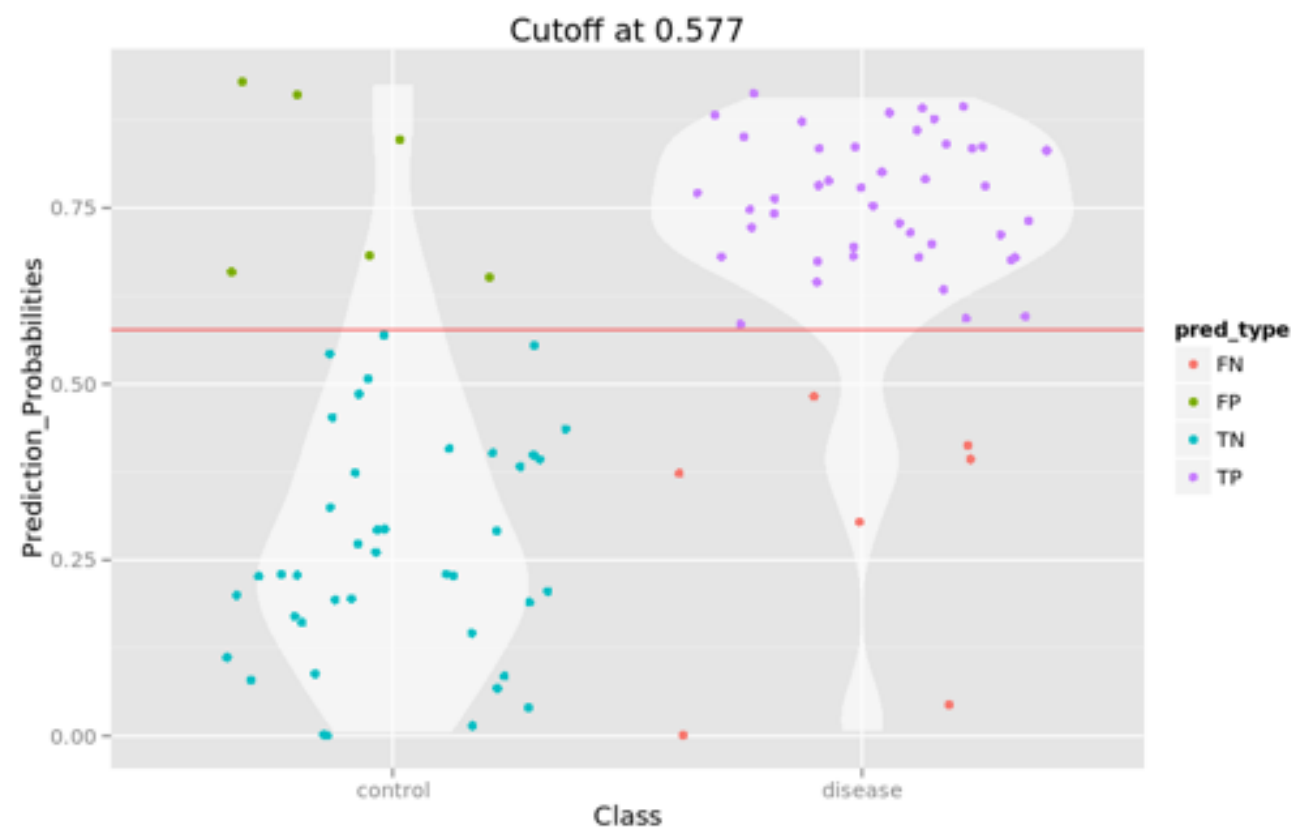

\section{Figure 4}

Violin plot showing the probability density of the data for the two compared classes, dependent on the previously obtained optimal cutoff on the corresponding ROC curve 


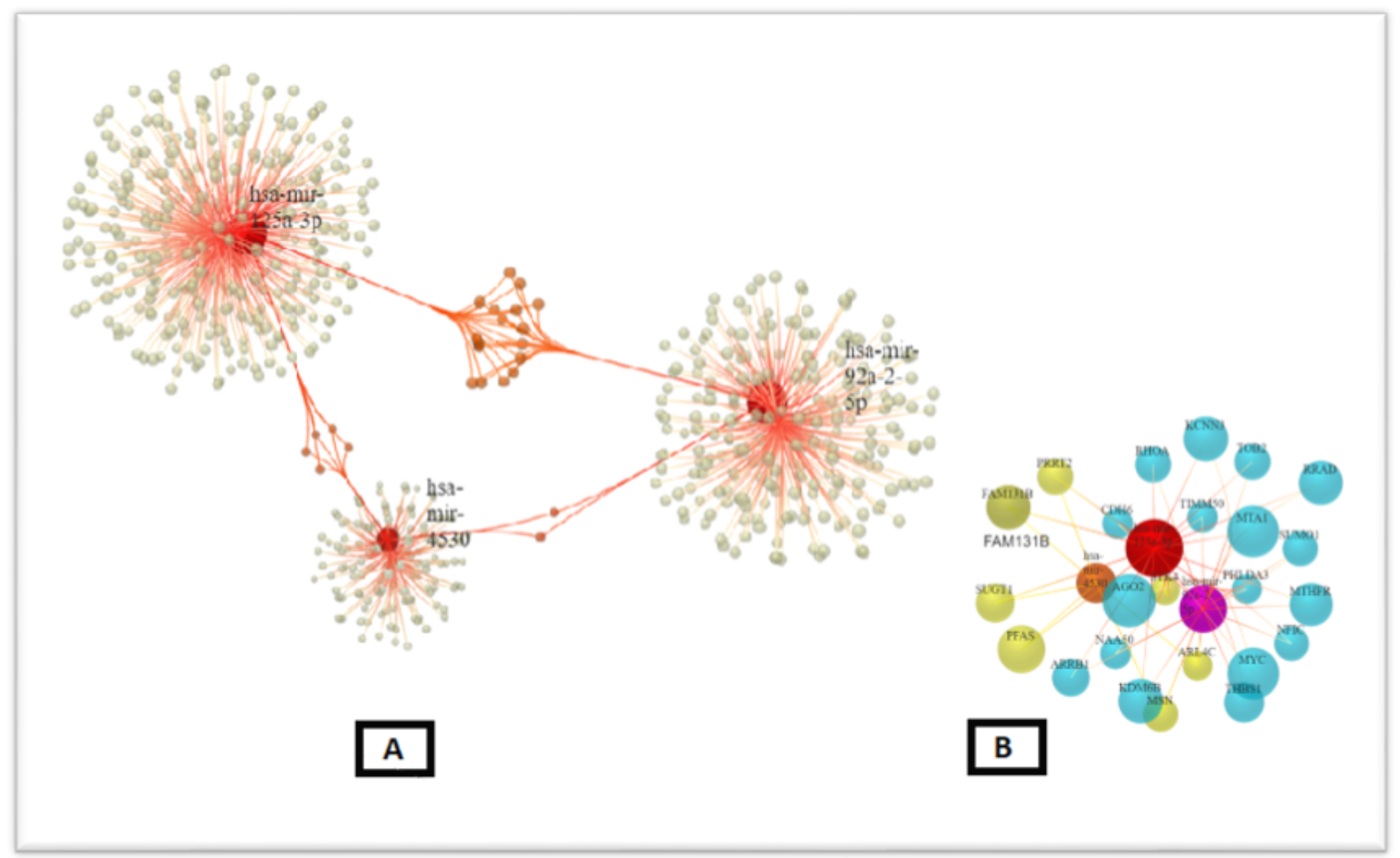

Figure 5

A: Regulatory network of miRNAs and predicted target genes. Red, Green and Orange circles represent the miRNAs, non-shared and shared target genes. B: The extracted module demonstrating the shared targets of considered miRNAs

\section{Supplementary Files}

This is a list of supplementary files associated with this preprint. Click to download.

- Supplementary.docx 